\title{
Increased susceptibility to oxidative damage in post-diabetic human myotubes
}

\author{
S. R. Costford - S. A. Crawford • R. Dent • \\ R. McPherson • M.-E. Harper
}

Received: 12 May 2009 / Accepted: 3 July 2009 / Published online: 16 August 2009

(C) Springer-Verlag 2009

\begin{abstract}
Aims/hypothesis Obesity is an important risk factor for the development of type 2 diabetes, but not all obese individuals develop this complication. The clinical signs of type 2 diabetes can often be reversed with weight loss; however, it is unknown whether the skeletal muscle oxidative stress associated with type 2 diabetes remains after weight loss. We hypothesised that chronic exposure to high glucose and insulin would re-elicit impaired metabolism in primary myotubes from patients with a history of type 2 diabetes.

Methods Obese participants with or without type 2 diabetes completed a standardised weight loss protocol, following which all participants were euglycaemic and had similar indices of insulin sensitivity. Satellite cells were isolated from muscle biopsies and differentiated under low or high glucose and insulin conditions (HGI).
\end{abstract}

S. R. Costford and S. A. Crawford contributed equally to this work.

Electronic supplementary material The online version of this article (doi:10.1007/s00125-009-1480-y) contains supplementary material, which is available to authorised users.

S. R. Costford · S. A. Crawford · M.-E. Harper $(\bowtie)$

Department of Biochemistry, Microbiology and Immunology,

Faculty of Medicine, University of Ottawa,

451 Smyth Road,

Ottawa, ON K1H 8M5, Canada

e-mail: mharper@uottawa.ca

R. Dent

Ottawa Hospital Weight Management Clinic,

Ottawa, ON, Canada

R. McPherson

Division of Cardiology, University of Ottawa Heart Institute,

Ottawa, ON, Canada
Results Cells from participants with no history of type 2 diabetes showed robust increases in mitochondrial content, citrate synthase and cytochrome $c$ oxidase activities when exposed to HGI. This increase in oxidative capacity was absent in cells from patients with a history of type 2 diabetes. High glucose and insulin caused increased oxidative damage in cells from the latter, despite higher superoxide dismutase expression. Cells from patients with a history of type 2 diabetes were unable to decrease mitochondrial membrane potential in response to HGI, potentially due to lower levels of uncoupling protein-3.

Conclusions/interpretation This is the first report to note that primary myotubes from patients with a history of type 2 diabetes are unable to adapt to a hyperglycaemichyperinsulinaemic challenge. We have demonstrated that impaired mitochondrial biogenesis and an inability to manage oxidative stress define a muscle phenotype at risk of obesity-associated type 2 diabetes.

Keywords Mitochondria - Obesity - Oxidative stress . Reactive oxygen species $\cdot$ Skeletal muscle $\cdot$ Type 2 diabetes mellitus · Uncoupling protein-3

$\begin{array}{ll}\text { Abbreviations } \\ \text { AU } & \text { Arbitrary units } \\ \text { DEXA } & \text { Dual energy x-ray absorptiometry } \\ \text { ETC } & \text { Electron transport chain } \\ \text { HGI } & \text { High glucose and insulin conditions } \\ \text { HNE } & \text { 4-Hydroxynonenal } \\ \text { IDV } & \text { Integrated density value } \\ \text { IMTG } & \text { Intramuscular triacylglycerol } \\ \text { LGI } & \text { Low glucose and insulin conditions } \\ \text { MnSOD } & \text { Manganese superoxide dismutase } \\ \text { ROS } & \text { Reactive oxygen species } \\ \text { SDH } & \text { Succinate dehydrogenase }\end{array}$


TMRE Tetramethylrhodamine, ethyl ester, perchlorate UCP3 Uncoupling protein-3

\section{Introduction}

Obesity is a major risk factor for several chronic diseases including cardiovascular disease, certain cancers and type 2 diabetes mellitus, as reviewed by Kopelman [1]. Among adults diagnosed with diabetes, $57 \%$ are obese and $28 \%$ are overweight [2]. Conversely, only $14 \%$ of obese adults have diabetes [3]. Thus while obesity is a well-recognised risk factor for type 2 diabetes, the relatively small percentage of obese individuals who also develop type 2 diabetes is consistent with the general knowledge that type 2 diabetes has a strong genetic component. However, despite recent advances, genetic and other determinants of risk for obesity-associated type 2 diabetes have not been fully elucidated [4].

One of the hallmarks of type 2 diabetes is insulin resistance. Normally, skeletal muscle accounts for $\sim 80 \%$ of postprandial glucose disposal, but in type 2 diabetes it becomes the primary site of insulin resistance [5]. The molecular mechanisms responsible for the progression of insulin resistance are still poorly understood. Ectopic accumulation of lipid in skeletal muscle (intramuscular triacylglycerol [IMTG]) is associated with obesity and highly correlated with the development of insulin resistance [6-8]. However, the observation that lean, insulin-sensitive endurance-trained athletes have high levels of IMTG has led to the hypothesis that altered or incomplete lipid metabolism may be an important factor in the progression of insulin resistance (reviewed by van Loon and Goodpaster [9]). Along these lines, it has been proposed that the overflow of triacylglycerol metabolites in skeletal muscle mitochondria leads to an uncoupling of the tricarboxylic acid cycle and the electron transport chain (ETC), resulting in an accumulation of incompletely oxidised long-chain fatty acids [10]. These may thus be diverted from the tricarboxylic acid cycle into other lipid metabolites such as ceramides, diacylglycerol and/or acylcarnitines, which in turn can interfere with the insulin signalling pathway [10, 11], as discussed in reviews [12-14]. Indeed, decreased mitochondrial content and impaired oxidative capacity are correlated with insulin resistance and associated with obesity [15-18], but effects can be mitigated by weight loss and physical activity $[19,20]$.

The delivery of a surfeit of metabolic substrates to mitochondria can lead to augmented reactive oxygen species (ROS) production and impaired mitochondrial function. ROS can cause lipid peroxidation, generating products such as methylcholanthrene and 4-hydroxynonenal (HNE), which can subsequently bind proteins, forming adducts and impair- ing protein function, as reviewed by Eriksson [21], Pieczenik and Neustadt [22], and Civitarese and Ravussin [23]. Oxidative stress has been implicated in the development of insulin resistance [24-28]; however the role of ROS in diabetic skeletal muscle is an emerging field. Recently, Chung et al. demonstrated that treatment of human skeletal muscle cells with high levels of hydrogen peroxide led to destruction of the insulin signalling pathway, but that pretreatment with troglitazone could prevent this ROS-induced insulin resistance [29]. The production of ROS by skeletal muscle and the mechanisms by which this tissue mitigates oxidative damage in the context of type 2 diabetes are areas of great interest.

The overall aim of the current preliminary study was to determine whether impaired metabolism could be detected in primary muscle cells of patients with a history of obesityassociated type 2 diabetes. Although no differences in oxidative capacity or oxidative damage were detected under standard low glucose and insulin conditions (LGI), cells from patients with a history of type 2 diabetes were unable to undergo mitochondrial biogenesis and had an increased susceptibility to oxidative damage when exposed to chronic high glucose and insulin conditions (HGI). These novel findings define a muscle phenotype at risk of obesityassociated type 2 diabetes and set the stage for future explorations.

\section{Methods}

Participants Test participants (hereafter referred to as postdiabetes mellitus participants) were obese and had a documented history of obesity-associated type 2 diabetes prior to weight loss. Inclusion criteria were: $\mathrm{BMI} \geq 30 \mathrm{~kg} /$ $\mathrm{m}^{2}$, fasting blood glucose $>7.0 \mathrm{mmol} / \mathrm{l}$ and $\mathrm{HbA}_{1 \mathrm{c}}>7 \%$, or treatment with hypoglycaemic agent(s) prior to weight loss. Age-, sex- and BMI-matched participants who had been similarly obese but normoglycaemic acted as non-diabetic controls. These non-diabetic participants had no family history of type 2 diabetes; however, two of the seven postdiabetes mellitus participants had at least one parent or sibling with type 2 diabetes. Physical activity was measured by questionnaire, which was adapted from a previous publication [30]. There were no differences in physical activity between non-diabetic and post-diabetes mellitus groups $(3.3 \pm 3.8 \mathrm{~h} /$ week and $4.6 \pm 3.2 \mathrm{~h} /$ week, respectively). Both populations underwent a 26 -week standard clinical weight loss programme at the Ottawa Hospital Weight Management Clinic (details, see Electronic supplementary material [ESM] and previous publications [31, 32]). Following weight loss, post-diabetes mellitus participants exhibited consistently normal blood glucose and $\mathrm{HbA}_{1 \mathrm{c}}$ levels $(\leq 6.1 \mathrm{mmol} / 1$ and $\leq 5.7 \%$, respectively). Some 
participants experienced a modest weight gain after completing the programme; however, all participants had had stable body weight for a minimum of 14 weeks prior to study (weight stability ranged from 14 to 102 weeks). Weight stability was defined as weight maintenance within $5 \%$ of body weight. Participant characteristics pre- and post-weight loss as well as at the time of study are described in Table 1.

All participants gave informed consent and these investigations were approved by the Human Research Ethics Committees of the Ottawa Hospital and the University of Ottawa Heart Institute.

Euglycaemic-hyperinsulinaemic clamp Insulin infusate was prepared in isotonic saline, to which $2 \mathrm{ml}$ of participant's blood was added per $50 \mathrm{ml}$ infusate to prevent absorption of insulin by glass/plastic. Regular insulin was diluted to $300 \mathrm{mU} / \mathrm{ml}$ in $0.9 \%$ saline (wt/vol.) and infused as 10 min priming infusion, followed by fixed infusion of
$40 \mathrm{mU} / \mathrm{m}^{2}$. Glucose was infused as $20 \%$ dextrose (wt/vol) and was started 4 min after initiation of insulin infusion at a rate of $2.0 \mathrm{mg} \mathrm{kg} \mathrm{min}^{-1}$ for 4 to $10 \mathrm{~min}$ and at $2.5 \mathrm{mg} \mathrm{kg}^{-1} \mathrm{~min}^{-1}$ for 10 to $15 \mathrm{~min}$. Blood samples were taken every $5 \mathrm{~min}$. Glucose infusion was adjusted to maintain plasma glucose at $5.0 \mathrm{mmol} / 1$ [33].

Body composition Whole-body fat mass, fat free mass and regional fat distribution were assessed by dual-energy x-ray absorptiometry (DEXA) (LUNAR Prodigy; GE Medical Systems, Madison, WI, USA). The precision of repeated measurements is expressed as the per cent coefficient of variation $(2.2 \%$ for fat mass percentage).

Muscle biopsies Biopsies of vastus lateralis were obtained from seven post-diabetes mellitus and seven non-diabetic participants using a $5 \mathrm{~mm}$ Bergstrom needle (Opitek, Glostrup, Denmark) as described previously [34]. Participants had refrained from physical activity for 3 days and had fasted
Table 1 Pre- and post-weight loss characteristics of postdiabetes mellitus and nondiabetic participants
Unless otherwise stated, values are means $\pm \mathrm{SD}, n=7$, Student's $t$ test for each variable (NS, except where noted)

${ }^{\text {a }}$ Blood glucose for postdiabetes mellitus was measured while on diet and pharmacological treatment for diabetes

${ }^{\mathrm{b}} \mathrm{HbA}_{1 \mathrm{c}}$ was not evaluated in non-diabetic participants prior to or during weight loss programme

${ }^{\mathrm{c}}$ The moderate weight gain in non-diabetic and postdiabetes mellitus groups occurred between the end of the weight loss programme (at week 26) and the beginning of the weight stability period (all participants had stable body weight for a minimum of 14 weeks prior to study)

${ }^{\mathrm{d}}$ Non-diabetic vs post-diabetes mellitus, $p<0.05$

N/A, not applicable

\begin{tabular}{|c|c|c|}
\hline Variables & Non-diabetic & Post-diabetes mellitus \\
\hline Age (years) & $52.1 \pm 11.0$ & $51.8 \pm 11.0$ \\
\hline Men/women $(n)$ & $6 / 1$ & $6 / 1$ \\
\hline Family history of type 2 diabetes & 0 & 2 \\
\hline No family history of type 2 diabetes & 7 & 5 \\
\hline \multicolumn{3}{|l|}{ Pre-weight loss } \\
\hline Body weight $(\mathrm{kg})$ & $144.7 \pm 30.3$ & $137.6 \pm 35.4$ \\
\hline BMI $\left(\mathrm{kg} / \mathrm{m}^{2}\right)$ & $47.6 \pm 9.7$ & $45.6 \pm 9.5$ \\
\hline Fasting blood glucose (mmol/l) & $5.7 \pm 0.76$ & $6.4 \pm 1.28^{\mathrm{a}}$ \\
\hline Fasting blood glucose range $(\mathrm{mmol} / \mathrm{l})$ & $4.3-6.8$ & $4.4-7.8^{\mathrm{a}}$ \\
\hline $\mathrm{HbA}_{1 \mathrm{c}}(\%)$ & $\mathrm{N} / \mathrm{A}^{\mathrm{b}}$ & $7.0 \pm 1.6$ \\
\hline Diabetes medication & 0 & 5 \\
\hline \multicolumn{3}{|l|}{ Post-weight loss at week 26} \\
\hline Weight $(\mathrm{kg})^{\mathrm{c}}$ & $104.9 \pm 21.9$ & $108.5 \pm 25.6$ \\
\hline BMI $\left(\mathrm{kg} / \mathrm{m}^{2}\right)$ & $34.5 \pm 6.8$ & $36.06 \pm 6.9$ \\
\hline Fasting blood glucose $(\mathrm{mmol} / \mathrm{l})$ & $5.03 \pm 0.72$ & $4.96 \pm 0.35$ \\
\hline $\mathrm{HbA}_{1 \mathrm{c}}(\%)$ & $\mathrm{N} / \mathrm{A}^{\mathrm{b}}$ & $5.2 \pm 0.4$ \\
\hline Diabetes medication & 0 & 0 \\
\hline \multicolumn{3}{|l|}{ At time of study } \\
\hline Time between week 26 and study (weeks) & $52.0 \pm 10.5$ & $50.8 \pm 10.4$ \\
\hline Duration of weight stability (weeks) & $31.7 \pm 31.8$ & $32.4 \pm 13.1$ \\
\hline Body weight $(\mathrm{kg})^{\mathrm{c}}$ & $117.5 \pm 20.0$ & $113.4 \pm 23.5$ \\
\hline BMI $\left(\mathrm{kg} / \mathrm{m}^{2}\right)$ & $38.7 \pm 6.4$ & $37.9 \pm 5.9$ \\
\hline Basal metabolic rate $(\mathrm{kJ} / \mathrm{day})$ & $9109 \pm 1406$ & $8392 \pm 1666$ \\
\hline Per cent fat-free mass (DEXA) & $61.8 \pm 6.0$ & $59.8 \pm 3.4$ \\
\hline Per cent fat mass (DEXA) & $39.6 \pm 3.41$ & $34.7 \pm 2.85^{\mathrm{d}}$ \\
\hline Per cent total body fat as truncal fat (DEXA) & $64.4 \pm 2.8$ & $58.5 \pm 4.7$ \\
\hline Fasting blood glucose (mmol/l) & $5.0 \pm 0.8$ & $4.9 \pm 0.5$ \\
\hline $\mathrm{HbA}_{1 \mathrm{c}}(\%)$ & $5.3 \pm 0.1$ & $5.4 \pm 0.2$ \\
\hline Diabetes medication & 0 & 0 \\
\hline
\end{tabular}


for $12 \mathrm{~h}$ prior to biopsy. A sample of muscle $(\sim 10 \mathrm{mg})$ was frozen in optimal cutting temperature compound for determination of fibre type ratio and IMTG. The remainder of muscle biopsy was processed for satellite cell isolation and culture.

Cell culture Freshly biopsied vastus lateralis was minced, subjected to trypsin digestion and plated in Ham's F-10 media supplemented with $15 \%$ fetal bovine serum (vol./vol.), $0.5 \mathrm{mg} / \mathrm{ml} \mathrm{BSA}, 1 \mu \mathrm{mol} / 1$ dexamethazone, $10 \mathrm{ng} / \mathrm{ml}$ EGF, $0.5 \mathrm{mg} / \mathrm{ml}$ fetuin and $0.25 \mathrm{pmol} / 1$ insulin. Muscle satellite cells were isolated using an immuno-based magnetic sorting technique as previously described [34]. Myoblasts were differentiated for 7 days prior to experimentation in LGI DMEM (5.5 mmol/l glucose, supplemented with $0.25 \mathrm{pmol} / 1$ insulin, $2 \%$ horse serum (vol./vol.), $1 \%$ antibiotic-antimycotic (vol./vol.) and $2.5 \mathrm{mg} / \mathrm{ml}$ gentamycin) or in HGI DMEM (25 mmol/l glucose, supplemented with $10 \mathrm{pmol} / \mathrm{l}$ insulin, $2 \%$ horse serum, $1 \%$ antibiotic-antimycotic and $2.5 \mathrm{mg} / \mathrm{ml}$ gentamycin).

Fibre type ratio Frozen tissues were sectioned and mounted on Superfrost slides (Fisher, Ottawa, ON, Canada). Type 1 and type 2 fibres were immunostained sequentially using A4.840 and N2.261 (Developmental Studies Hybridoma Bank, Iowa City, IA, USA) primary antibodies and biotinylated goat anti-mouse (BA-9200; Vector Laboratories, Burlington, ON, Canada) secondary antibody. Type 1 fibres were stained red (SK5100; Vector Laboratories) and type 2 fibres were stained blue (SK5300; Vector Laboratories). The number of type 1 and type 2 fibres was counted in three fields of view in each of three sections per participant. The fibre type ratio was determined from these numbers.

Intramuscular triacylglycerol IMTG content was evaluated ex vivo and in vitro. Ex vivo determinations were conducted as described previously [34]. Frozen tissues were sectioned, mounted on Superfrost slides and fixed in $10 \%$ neutral buffered formalin (vol./vol.). Sections were stained with $1 \%$ osmium tetroxide (wt/vol.) and counterstained with haematoxylin and eosin. Three fields of view in each of three sections per participant were quantified using Northern Eclipse imaging software (Empix Imaging, Mississauga, ON, Canada) to determine the amount of IMTG relative to the cross-sectional fibre area.

For in vitro analyses, differentiated myotubes were suspended in $25 \mathrm{mmol} / 1$ Tris-HCl pH 7.5 with $1 \mathrm{mmol} / \mathrm{l}$ EDTA. Lipids were extracted via chloroform-methanol $(2: 1)$, evaporated under $\mathrm{N}_{2}$ gas and dissolved in 2-propanol. Triacylglycerol concentration was determined spectrophotometrically (L-Type TG H; Wako, Richmond, VA, USA) and normalised to protein content as determined by bicinchoninic acid assay.
Glycogen Differentiated myotubes were suspended in PBM buffer $\left(20 \mathrm{mmol} / 1 \mathrm{KH}_{2} \mathrm{PO}_{4}, 10 \mu \mathrm{mol} / 1 \mathrm{CaCl}_{2}, 1 \mathrm{mmol} / 1 \mathrm{MgCl}_{2}\right.$, pH6.1) and lysed by freeze-thaw. Samples were boiled for $20 \mathrm{~min}$ in $30 \% \mathrm{KOH}$ (wt/vol.) saturated with anhydrous $\mathrm{Na}_{2} \mathrm{SO}_{4}$. Glycogen was precipitated with $95 \%$ ethanol (vol/vol.), dissolved in double-distilled $\mathrm{H}_{2} \mathrm{O}$ and incubated at $100^{\circ} \mathrm{C}$ for $20 \mathrm{~min}$ with $0.2 \%$ anthrone (wt/vol.) in $\mathrm{H}_{2} \mathrm{SO}_{4}$. Glycogen concentration was determined spectrophotometrically by measuring absorbance at $620 \mathrm{~nm}$ relative to an oyster glycogen standard curve, and normalised to protein content.

Mitochondrial enzyme activities Intact mitochondria were isolated from differentiated myotubes using a mitochondrial isolation kit (MITOISO2; Sigma, St Louis, MO, USA). Mitochondrial yield was expressed as mitochondrial protein content per cellular protein content. Cytochrome $c$ oxidase activity was assayed in intact mitochondria using a kit (Cytocox1; Sigma). Citrate synthase activity was determined in lysed mitochondria using a kit (CS0720; Sigma). Enzyme activities were normalised to mitochondrial and cellular protein contents.

Mitochondrial membrane potential Myoblasts were seeded at 20,000 cells/well in 96-well plates and differentiated in LGI/HGI media for 7 days. Cells were incubated with $800 \mathrm{nmol} / 1$ tetramethylrhodamine, ethyl ester (TMRE) perchlorate in either LGI-PBS $(5.5 \mathrm{mmol} / 1$ glucose, $0.25 \mathrm{pmol} / 1$ insulin) or HGI-PBS $(25 \mathrm{mmol} / 1$ glucose, $10 \mathrm{pmol} / 1 \mathrm{insulin})$. Incubation was for $15 \mathrm{~min}$ at $37^{\circ} \mathrm{C}$ in the dark. Control wells contained no cells. Fluorescence was measured $15 \mathrm{~min}$ post-incubation (excitation $544 \mathrm{~nm}$, emission $590 \mathrm{~nm}$ ). TMRE is a lypophilic cationic dye that accumulates in the mitochondria proportional to membrane potential and through mitochondrial accumulation quenches TMRE fluorescence [35]. Membrane potential was expressed as the absolute value of the decrease in TMRE fluorescence and normalised to mitochondrial protein content.

Western blotting Electrophoresis of cell lysates or isolated mitochondria was carried out on a $10 \%$ polyacrylamide gel (wt/vol.). Proteins were then transferred to a nitrocellulose membrane. Primary antibodies were: uncoupling protein-3 (UCP3) (ab-3477; Abcam, Cambridge, MA, USA), $\beta$-actin (4967; Cell Signaling, Danvers, MA, USA), manganese superoxide dismutase (MnSOD) and succinate dehydrogenase (SDH) (sc-30080 and sc-59687, respectively; Santa Cruz Biotechnology, Santa Cruz, CA, USA). Secondary antibodies were: goat anti-rabbit horseradish peroxidase and goat anti-mouse horseradish peroxidase (sc-2030 and sc-2031, respectively; Santa Cruz). Visualisation was achieved using an enhanced chemiluminescence kit 
(Amersham Pharmacia, Baie d'Urfe, QC, Canada). Spot densitometry was performed using an Alpha multi-image light camera and Alpha imaging software (Alpha Imaging, Willoughby, $\mathrm{OH}, \mathrm{USA}$ ). Values represent the integrated density value (IDV) of UCP3 or MnSOD divided by the IDV of $\beta$-actin or SDH.

HNE-histidine adducts HNE-histidine adduct content was determined by ELISA (STA-334; Cell Biolabs, San Diego, CA, USA). Protein samples were absorbed on to a 96-well ELISA plate in triplicate for $2 \mathrm{~h}$ at $37^{\circ} \mathrm{C}$. Wells were probed with an anti-HNE-histidine antibody and horseradish peroxidase-conjugated secondary antibody. Absolute HNE-histidine adduct content was determined spectrophotometrically by comparing samples with a standard curve of HNE-BSA standards at $600 \mathrm{~nm}$.

Statistical methods A Student's $t$ test was used to assess statistical differences between clinical variables as well as differences in fibre type percentage, MnSOD expression and UCP3 expression. A one-way ANOVA with Tukey's post-test was used to assess statistical differences in IMTG content, glycogen content, mitochondrial yield, citrate synthase activity, cytochrome $c$ oxidase activity, HNEhistidine adducts and mitochondrial membrane potential (Figs 1, 2, 3 and 4). Confidence intervals were set at 95\%.
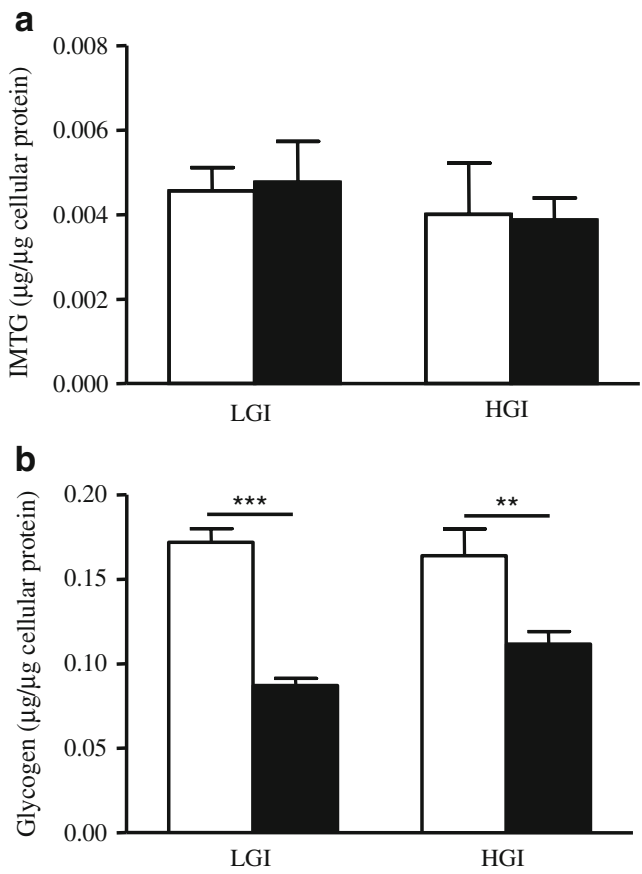

Fig. 1 Fuel storage. IMTG (a) and glycogen (b) normalised to cellular protein in myotubes from non-diabetic participants (white bars) and post-diabetes mellitus participants (black bars) differentiated under LGI or HGI. Values are means $\pm \mathrm{SD}, n=3$ (a), $n=5$ (b). One-way ANOVA, Tukey's post-test, NS (a), ${ }^{* *} p<0.01,{ }^{* * *} p<0.001$ (b)

\section{Results}

Study participants Prior to weight loss, all post-diabetes mellitus participants met the American Diabetes Association criteria for diabetes [36] or were taking oral glucoselowering agents. Following weight loss, post-diabetes mellitus participants became normoglycaemic and remained so at the time of study. Non-diabetic participants did not meet the criteria for diabetes before or after weight loss (mean fasting glucose $5.7 \pm 0.76 \mathrm{mmol} / \mathrm{l}$ ). At the time of study, the two groups showed no differences in fasting blood glucose, $\mathrm{HbA}_{1 \mathrm{c}}$ or fat-free mass; however, the nondiabetic group had a slightly higher percentage of body fat (Table 1). As assessed by a euglycaemic-hyperinsulinaemic clamp, participant groups demonstrated similar insulinstimulated glucose disposal rates and insulin sensitivity indices (Table 2). No differences were detected in the insulin sensitivity of primary myotubes from non-diabetic compared with post-diabetes mellitus participants, as assessed by insulin-stimulated glucose uptake and insulinstimulated glycogen synthesis (ESM Fig. 1).

Fibre type ratio No differences were observed in the fibre type distribution between the non-diabetic and the postdiabetes mellitus populations (Fig. 5). The fibre type distribution for both was approximately $40 \%$ type 1 and $60 \%$ type 2 .

Fuel storage Similarly, there were no differences in ex vivo IMTG content between non-diabetic and post-diabetes mellitus participants $(63.78 \pm 6.58$ arbitrary units [AU] vs $71.79 \pm 12.18 \mathrm{AU}$, respectively). IMTG content was also assessed in vitro in myotubes differentiated in standard LGI conditions or in 'diabetes-like' HGI conditions. Surprisingly, no differences were detected in IMTG content between LGI and HGI conditions in post-diabetes mellitus or nondiabetic participants. There were also no differences in IMTG content between post-diabetes mellitus and nondiabetic participants in either condition (Fig. 1a). Postdiabetes mellitus myotubes exhibited $\sim 50 \%$ lower intracellular glycogen levels than those of non-diabetic participants in LGI $(p<0.01)$ and $\sim 30 \%$ lower levels of glycogen in HGI (Fig. 1b).

Mitochondrial content and oxidative capacity No differences were seen in mitochondrial content between nondiabetic and post-diabetes mellitus myotubes under LGI conditions; however, mitochondrial content was nearly doubled in non-diabetic myotubes when exposed to HGI $(p<0.01)$, whereas no increase was observed in post-diabetes mellitus participants under the same conditions (Fig. 2a).

Cellular citrate synthase activity was assessed as a marker of mitochondrial content. Non-diabetic myotubes 
Fig. 2 Oxidative capacity.

(a) Mitochondrial yield as mitochondrial (mito) protein content per cellular protein content, (b) citrate synthase activity normalised to cellular protein, (c) citrate synthase activity normalised to mitochondrial protein, (d) cytochrome $c$ oxidase activity normalised to cellular protein and (e) cytochrome $c$ oxidase activity normalised to mitochondrial protein in (a-e) myotubes from non-diabetic participants (white bars) and post-diabetes mellitus participants (black bars) that had been differentiated under LGI or HGI. Values are means $\pm \mathrm{SD}, n=3$, one-way ANOVA, Tukey's post-test, $* p<0.05, * * p<0.01$, $* * * p<0.001$ a

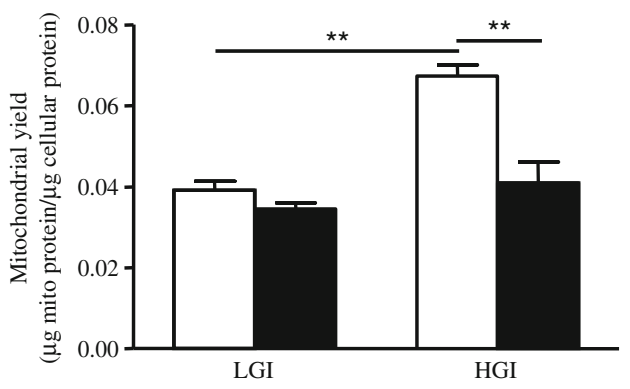

b

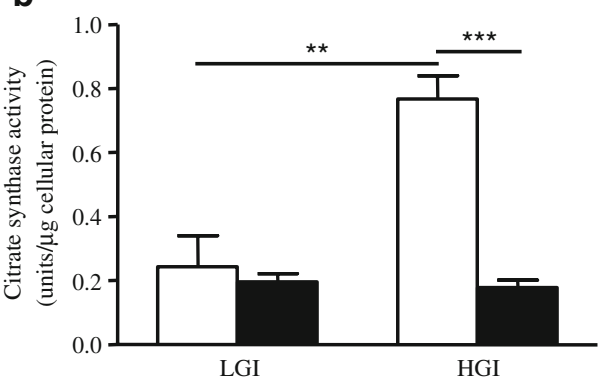

C

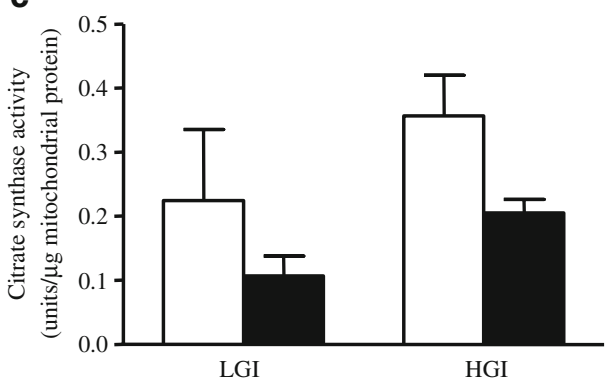

d

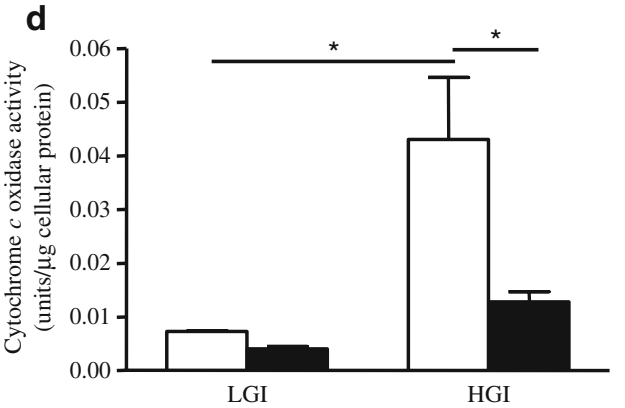

e

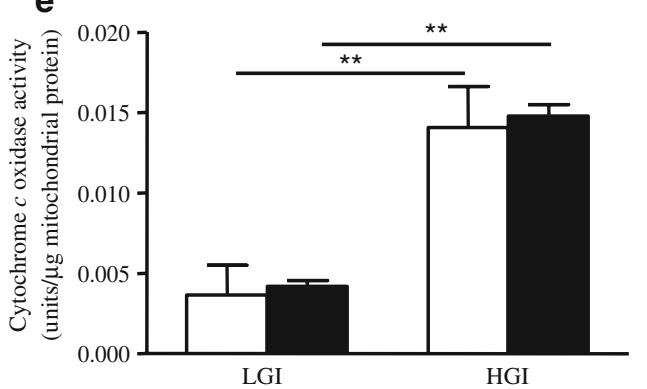

showed an overall increase of $\sim 65 \%$ in citrate synthase activity when exposed to HGI $(p<0.01)$, whereas postdiabetes mellitus myotubes exposed to HGI showed no differences in citrate synthase activity (Fig. 2b). When normalised to mitochondrial content, there was a trend for post-diabetes mellitus myotubes to have lower citrate

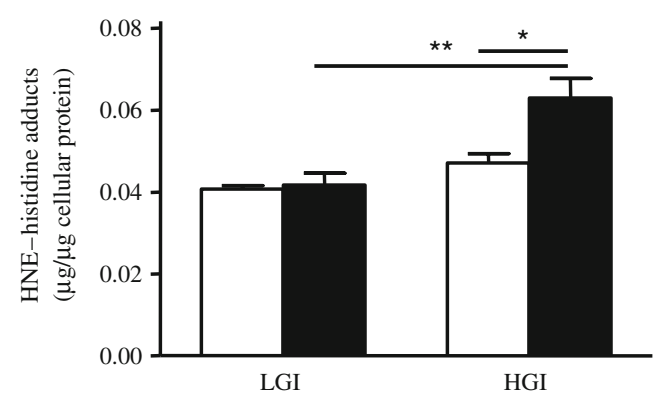

Fig. 3 Oxidative damage. HNE-histidine adducts normalised to cellular protein content. Myotubes from non-diabetic participants (white bars) and post-diabetes mellitus participants (black bars) were differentiated under LGI or HGI. Values are means $\pm \mathrm{SD}, n=4$, oneway ANOVA, Tukey's post-test, ${ }^{*} p<0.05,{ }^{* *} p<0.01$ synthase activity than non-diabetic myotubes in LGI and HGI, although this did not reach statistical significance (Fig. 2c). These data suggest that the lack of an HGIinduced increase in citrate synthase activity in post-diabetes mellitus myotubes is due mainly to the lack of mitochondrial biogenesis under these conditions.

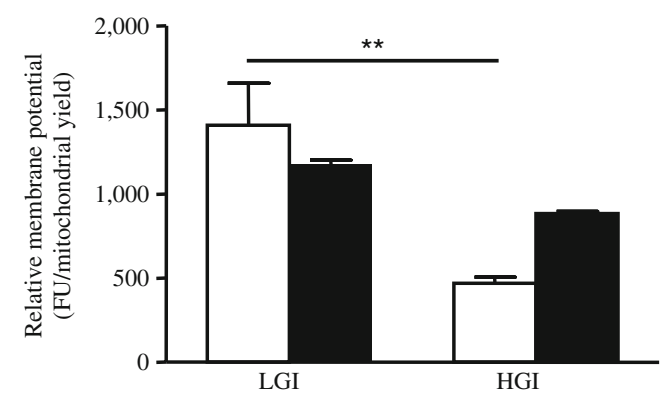

Fig. 4 Mitochondrial membrane potential. Relative mitochondrial membrane potential expressed in fluorescence units (FU) normalised to mitochondrial yield. Myotubes from non-diabetic participants (white bars) and post-diabetes mellitus participants (black bars) were differentiated under LGI or HGI. Values are means $\pm \mathrm{SD}, n=4$, oneway ANOVA, Tukey's post-test, ${ }^{* *} p<0.01$ 
Table 2 Post-weight loss measurements taken during the euglycaemic-hyperinsulinaemic clamp

Variables $\quad$ Non-diabetic participants

Post-diabetes mellitus participants

Baseline blood glucose (mmol/l)

$5.67 \pm 0.63$

$47.6 \pm 33.6$

$1.66 \pm 2.86$

$1071 \pm 413$

60-120 min insulin (pmol/1)

Insulin-stimulated glucose disposal ( $\left.\mathrm{mmol} \mathrm{kg}^{-1} \mathrm{~min}^{-1}\right)$ Insulin sensitivity $\left(M / \mathrm{I}_{\text {clamp }}\right)\left(\mathrm{nmol} \mathrm{kg} \mathrm{kin}^{-1} \mathrm{pmol}^{-1}\right)$
$0.272 \pm 0.103$

$319.8 \pm 232.0$
$5.40 \pm 0.71$
$70.0 \pm 21.4$
$2.32 \pm 4.24$
$1002 \pm 222$
$0.262 \pm 0.132$
$282.5 \pm 180.7$

Values are means $\pm \mathrm{SD}, n=7$, Student's $t$ test for each variable (NS)

$\mathrm{I}_{\text {clamp }}$, euglycaemic-hyperinsulinaemic clamp (as described in the Methods; see also DeFronzo et al. [33])

Cellular cytochrome $c$ oxidase activity was used as a second marker of mitochondrial content. When normalised to cellular protein, non-diabetic myotubes showed an overall increase of $\sim 80 \%$ in cytochrome $c$ oxidase activity when exposed to HGI conditions $(p<0.05)$. Post-diabetes mellitus myotubes, in contrast, did not show a significant increase in overall cytochrome $c$ oxidase activity (Fig. 2d). When normalised to mitochondrial content, non-diabetic and post-diabetes mellitus myotubes showed an increase in cytochrome $c$ oxidase activity in HGI conditions compared with LGI conditions $(p<0.01)$, indicating an upregulation of ETC capacity per mitochondrion (Fig. 2e). There were no differences in ETC capacity between non-diabetic and

a

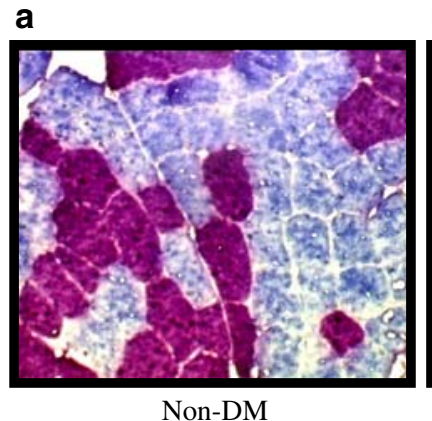

b

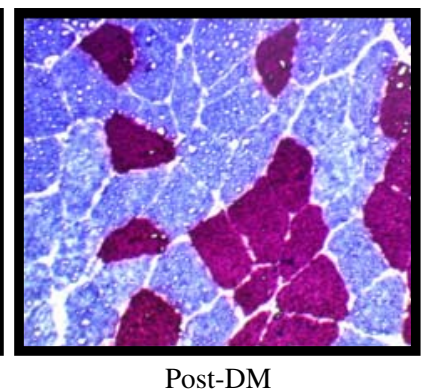

C

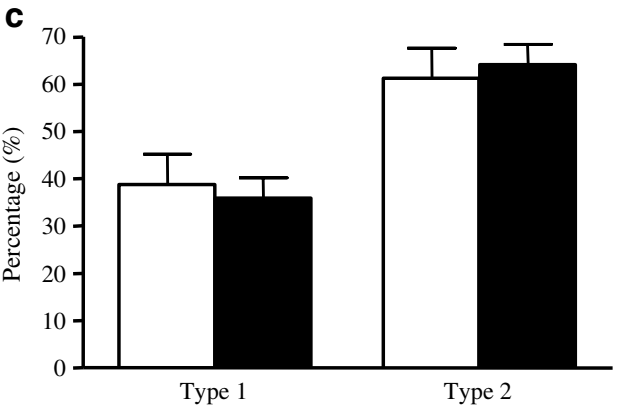

Fig. 5 Fibre type ratio. Type 1 fibres stained red and type 2 fibres stained blue in representative muscle sections of (a) a non-diabetic (Non-DM) and (b) a post-diabetes mellitus (Post-DM) participant. c Fibre type ratio expressed as a percentage. Values are means \pm SD, $n=6$, Student's $t$ test for each fibre type, NS. White bars, non-diabetic participants; black bars, post-diabetes mellitus participants post-diabetes mellitus myotubes in LGI or HGI conditions at the level of the mitochondrion. Altogether, these data indicate that due to higher mitochondrial content, overall oxidative capacity is higher in non-diabetic myotubes than in post-diabetes mellitus myotubes, when these are exposed to HGI conditions.

Oxidative damage and mitochondrial membrane potential Oxidative damage was assessed by measuring HNE-protein adducts. There were no differences in HNE-protein adduct content in non-diabetic and post-diabetes mellitus myotubes under LGI conditions; however, when myotubes were differentiated in HGI conditions, a significant increase was observed in HNE-adduct content in the post-diabetes mellitus group $(p<0.01)$, but not in the non-diabetic group (Fig. 3).

In response to higher ROS production, genes involved in antioxidant pathways are typically upregulated. Levels of MnSOD, the mitochondrial form of superoxide dismutase, were found to be higher in post-diabetes mellitus myotubes when differentiated under LGI $(p<0.05)$ and HGI $(p<0.01)$ (Fig. 6), indicating that although higher oxidative damage was not observed in post-diabetes mellitus cells under LGI conditions, it is likely that higher amounts of ROS were being produced.

Lower rates of ROS production have been associated with lower mitochondrial membrane potential. The mitochondrial membrane potential in non-diabetic myotubes was decreased in HGI compared with LGI $(p<0.01)$, but this was not observed in post-diabetes mellitus myotubes (Fig. 4). It has been hypothesised that UCP3 may mitigate ROS production by uncoupling oxidative phosphorylation in situations of high membrane potential [37-39]. No overall differences in UCP3 protein content were observed between post-diabetes mellitus and non-diabetic myotubes under LGI conditions (Fig. 7a, c). However, upon exposure to HGI conditions, post-diabetes mellitus myotubes exhibited threefold lower levels of UCP3 $(p<0.05)$ (Fig. 7b, d). When UCP3 was normalised to mitochondrial content, post-diabetes mellitus participants had $\sim 35 \%$ less 
Fig. 6 MnSOD content. a, b Representative western blots of MnSOD and SDH loading controls. Mitochondria derived from non-diabetic (Non-DM)/postdiabetes mellitus (Post-DM) myotubes were differentiated under (a) LGI and (b) HGI. c Quantification of MnSOD content with IDV of MnSOD normalised to SDH IDV for LGI and (d) for HGI. Values are means $\pm \mathrm{SD}, n=4$, Student's $t$ test, ${ }^{*} p<0.05, * * p<0.01$ a
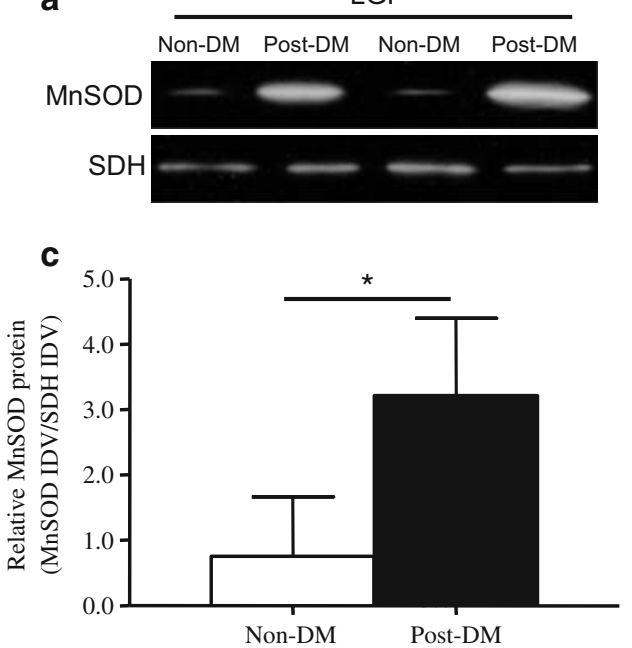

b
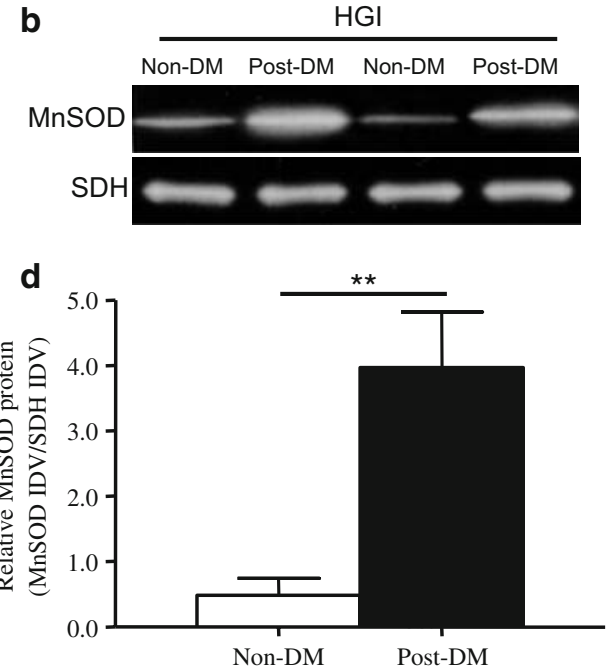

UCP3 than non-diabetic participants $(p<0.05)$, indicating decreased levels of UCP3 per mitochondrion (Fig. 7f).

\section{Discussion}

Weight loss through restriction of energy intake improves glycaemic control in patients with type 2 diabetes [40, 41].
In order to better understand the mechanistic associations between obesity and type 2 diabetes, and to avoid the confounding effects of concurrent hyperglycaemia or diabetes medications, we compared the skeletal muscle characteristics of participants with or without a history of obesity-associated diabetes, all of whom were similarly euglycaemic following weight loss.

IMTG is best correlated with an individual's degree of insulin resistance, regardless of BMI [6-8]. No differences
Fig. 7 UCP3 content. a, b Representative western blots of UCP3 and $\beta$-actin loading controls using lysates from nondiabetic (Non-DM)/postdiabetes mellitus (Post-DM) myotubes that had been differentiated under (a) LGI and (b) HGI. (c) Quantification of UCP3 content as IDV of UCP3 normalised to $\beta$-actin IDV for LGI and (d) HGI. (e) UCP3 content normalised to mitochondrial yield for LGI and (f) for HGI. Values are means $\pm \mathrm{SD}$, $n=4$, Student's $t$ test, ${ }^{*} p<0.05$, $* * * p<0.001$ a
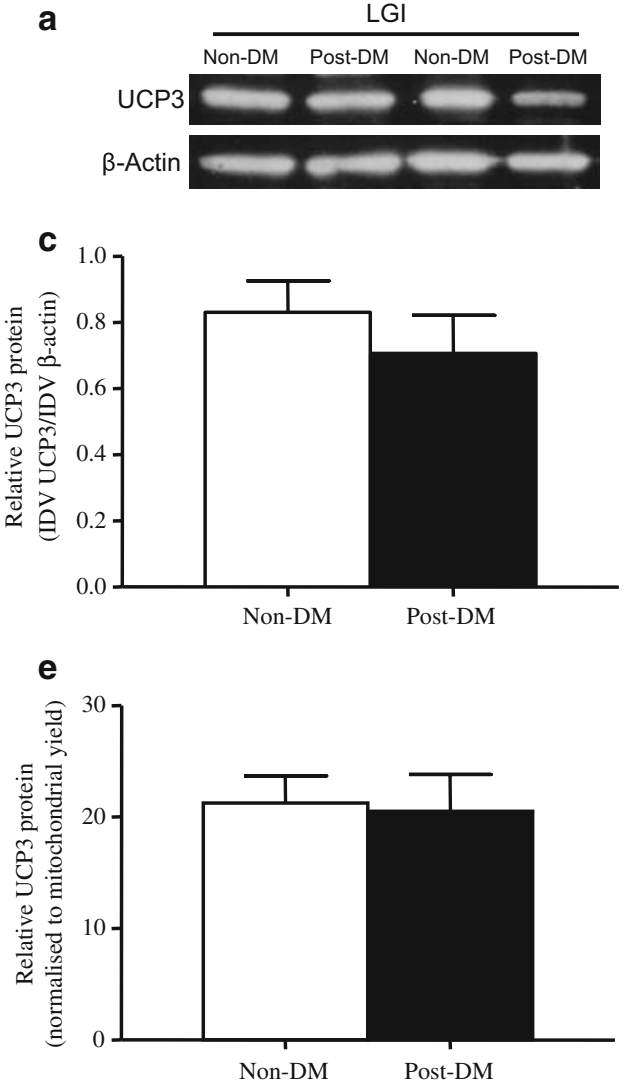

b

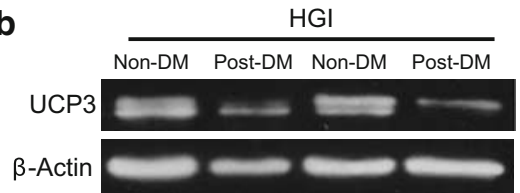

d

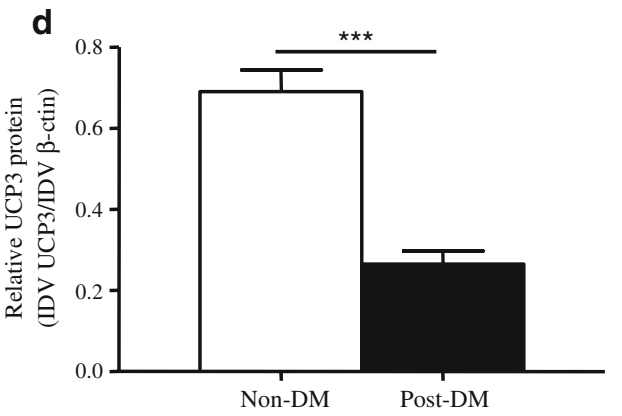

f

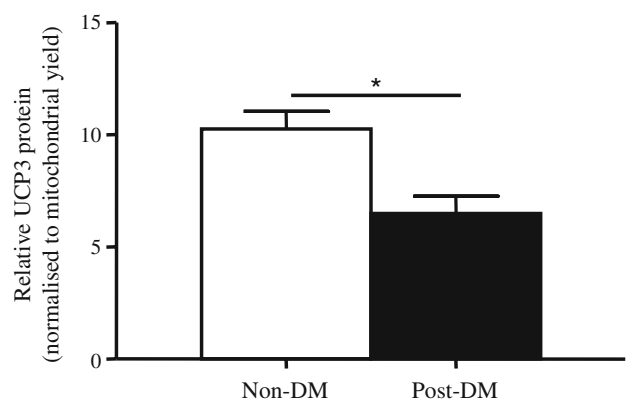


in IMTG were observed in LGI or HGI, indicating that hyperglycaemia-hyperinsulinaemia is not sufficient to elicit higher levels of IMTG in myotubes of post-diabetes mellitus participants. Conversely, glycogen levels in postdiabetes mellitus myotubes were lower in LGI and HGI. These results are consistent with reports that overall muscle glycogen content is lower in individuals at risk of type 2 diabetes as well as in those who have the disease [42, 43]. Interestingly, however, glycogen levels were lower in postdiabetes mellitus cells, although no differences in insulin sensitivity were seen between non-diabetic and postdiabetes mellitus cells. Glycogen deficit may therefore be an early marker of dysfunctional metabolism in muscle of individuals prone to obesity-associated diabetes or perhaps an imprint left in the satellite cells of individuals who have had type 2 diabetes, despite reversal of clinical symptoms of the disease via weight loss.

It has been previously shown that genes involved in oxidative metabolism and mitochondrial biogenesis are downregulated in skeletal muscle of individuals with type 2 diabetes, in individuals who are insulin-resistant and in healthy offspring of type 2 diabetes patients [16]. Functional impairment of skeletal muscle mitochondria has also been shown in these populations $[18,44]$. Our data suggest that, in individuals prone to obesity-associated diabetes, the defect in oxidative metabolism lies in the inability of myotubes to undergo mitochondrial biogenesis in response to HGI. Although overall impaired induction of citrate synthase and cytochrome $c$ oxidase activities was observed in postdiabetes mellitus cells, these deficiencies can be accounted for by the lack of mitochondrial biogenesis. Cytochrome $c$ oxidase activity was upregulated per mitochondrion in nondiabetic and post-diabetes mellitus participants when exposed to HGI, indicating that no impairment in ETC function exists at the level of the mitochondrion. These results indicate that the re-eliciting of impaired oxidative metabolism in cells from post-diabetes mellitus participants exposed to HGI is due to impaired stimulus of mitochondrial biogenesis.

Increased oxidative stress has been closely associated with insulin resistance and diabetes in a number of tissues, including skeletal muscle and adipose, as reviewed by Unoki and Yamagishi [45]. We found no differences in oxidative damage between non-diabetic and post-diabetes mellitus myotubes under LGI, but did see increased oxidative damage in post-diabetes mellitus myotubes under HGI. Taken alone, this result suggests that increased ROS production occurred under HGI, but post-diabetes mellitus cells were unable to mitigate that increase. However, the fact that MnSOD protein levels were increased in post-diabetes mellitus cells under LGI and HGI suggests that ROS production was increased under both conditions and that this antioxidant system was sufficient to prevent ROS damage in these cells under LGI, but not under HGI. To explore a possible mechanism that could explain the differences in oxidative damage between post-diabetes mellitus and non-diabetic cells, we measured mitochondrial membrane potential. The decrease in membrane potential that was observed in non-diabetic cells exposed to HGI may explain their resistance to oxidative damage. Post-diabetes mellitus cells were not able to lower their membrane potential in the face of increased reducing equivalents entering the ETC (i.e. under HGI) and therefore ROS production was augmented.

There are at least two possible explanations for the underlying mechanism of decreased mitochondrial membrane potential in non-diabetic cells but not in post-diabetes mellitus cells exposed to chronic HGI. The decrease in membrane potential in non-diabetic cells can at least be partially explained by the HGI-induced increase in mitochondrial content. With an $\sim 85 \%$ increase in mitochondrial content, the non-diabetic cells would be well equipped to metabolise the reducing equivalents provided by increased glycolytic flux, unlike the post-diabetes mellitus cells, which had no increase in mitochondrial content. The robust increase in oxidative capacity seen in non-diabetic cells may explain the resultant drop in mitochondrial membrane potential: substrates would be distributed among almost double the amount of mitochondria. With fewer reducing equivalents being delivered to the ETC per mitochondrion, lower membrane potential would ensue.

The mechanisms underlying the absence of a decrease in membrane potential in post-diabetes mellitus cells are likely to be multi-factorial. A second possible explanation relates to ROS signalling within the mitochondrion. High glucose exposure (i.e. more substrates being delivered to cells) would initially result in more reducing equivalents being delivered to the ETC per mitochondrion, driving up mitochondrial membrane potential and increasing ROS production. HNE can then be produced by ROS-lipid interaction. UCP3 is a mitochondrial inner membrane protein with unresolved physiological function(s). However, it has been hypothesised to mediate a proton leak when activated by HNE, reducing mitochondrial membrane potential and mitigating production of ROS in a negative feedback loop [39]. Interestingly, there were no differences in UCP3 expression between nondiabetic and post-diabetes mellitus participants under LGI conditions, but post-diabetes mellitus cells had lower expression of UCP3 at both the cellular and mitochondrial levels when exposed to HGI. These results corroborate previous reports of decreased UCP3 expression documented in vastus lateralis of individuals with type 2 diabetes [46], but now link decreased UCP3 expression to failed membrane potential response and increased oxidative damage under HGI. Thus, although the HNE signal is being generated in post-diabetes mellitus cells, it appears to be unable to activate UCP3 to effect a lower membrane potential and ultimately decrease ROS production. 
One of the limitations of this study was that individuals participating in the weight loss protocol had BMIs in the class III obesity range $\left(\geq 40 \mathrm{~kg} / \mathrm{m}^{2}\right)$ prior to weight loss. Following weight loss, they had BMIs in the class II obesity range $\left(35-39.9 \mathrm{~kg} / \mathrm{m}^{2}\right)$ (Table 1). Since the metabolic characteristics of primary myotubes from extremely obese participants may differ from those of obese participants [47], the conclusions drawn from this study may be limited to the population studied. Another limitation was the varying duration of the participants' weight stability prior to biopsy. However, the effect of interparticipant weight stability variability did not affect the clear differences in myotube metabolism observed between non-diabetic and post-diabetes mellitus donors.

Overall, this preliminary study has shown that impaired metabolism can be elicited in muscle cells from obese euglycaemic participants with a history of obesity-associated diabetes, despite resolution of the clinical diabetic state through weight loss. We have documented defects in mitochondrial biogenesis as well as the inability of muscle cells to mitigate ROS production and oxidative damage in response to situations of chronic HGI. These results provide new insight into the metabolic differences between individuals who are prone to obesity-associated diabetes and those who are not. However, it should be noted that these results do not necessarily apply to lean individuals at risk of type 2 diabetes. Although this study has provided clues as to the mechanisms of perturbed metabolism, it remains to be determined whether these phenotypic characteristics are genetically programmed or acquired epigenetically.

Acknowledgements Financial support for this study was provided by grants from the Heart and Stroke Foundation of Ontario (NA-5413; to R. McPherson and M.-E. Harper) and the Canadian Institutes of Health Research: Institute of Nutrition Metabolism and Diabetes (57810; to M.-E. Harper). The authors would like to thank E. Doucet (School of Human Kinetics, University of Ottawa) for assistance with DEXA, and H. Doelle (University of Ottawa Heart Institute) and B. Bradley (Ottawa Hospital Weight Management Clinic) for assistance with clinical investigations. We also thank L. Jui (Department of Biochemistry, Microbiology and Immunology, University of Ottawa) for assistance with histology and S. Chaudhry (Department of Biochemistry, Microbiology and Immunology, University of Ottawa) for early biopsy processing and analysis of histology.

Duality of interest The authors declare that there is no duality of interest associated with this manuscript.

\section{References}

1. Kopelman PG (2000) Obesity as a medical problem. Nature 404:635-643

2. Gregg EW, Cheng YJ, Narayan KM, Thompson TJ, Williamson DF (2007) The relative contributions of different levels of overweight and obesity to the increased prevalence of diabetes in the United States: 1976-2004. Prev Med 45:348-352
3. Gregg EW, Cheng YJ, Cadwell BL et al (2005) Secular trends in cardiovascular disease risk factors according to body mass index in US adults. JAMA 293:1868-1874

4. Frayling TM (2007) Genome-wide association studies provide new insights into type 2 diabetes aetiology. Nat Rev Genet 8:657-662

5. Ferrannini E, Smith JD, Cobelli C, Toffolo G, Pilo A, DeFronzo RA (1985) Effect of insulin on the distribution and disposition of glucose in man. J Clin Invest 76:357-364

6. Phillips DI, Caddy S, Ilic V et al (1996) Intramuscular triglyceride and muscle insulin sensitivity: evidence for a relationship in nondiabetic subjects. Metabolism 45:947-950

7. Perseghin G, Scifo P, De Cobelli F et al (1999) Intramyocellular triglyceride content is a determinant of in vivo insulin resistance in humans: a $1 \mathrm{H}-13 \mathrm{C}$ nuclear magnetic resonance spectroscopy assessment in offspring of type 2 diabetic parents. Diabetes 48:1600-1606

8. Krssak M, Falk Petersen K, Dresner A et al (1999) Intramyocellular lipid concentrations are correlated with insulin sensitivity in humans: a $1 \mathrm{H}$ NMR spectroscopy study. Diabetologia 42:113-116

9. van Loon LJ, Goodpaster BH (2006) Increased intramuscular lipid storage in the insulin-resistant and endurance-trained state. Pflugers Arch 451:606-616

10. Koves TR, Ussher JR, Noland RC et al (2008) Mitochondrial overload and incomplete fatty acid oxidation contribute to skeletal muscle insulin resistance. Cell Metab 7:45-56

11. Hegarty BD, Cooney GJ, Kraegen EW, Furler SM (2002) Increased efficiency of fatty acid uptake contributes to lipid accumulation in skeletal muscle of high fat-fed insulin-resistant rats. Diabetes 51:1477-1484

12. Muoio DM, Newgard CB (2008) Mechanisms of disease: molecular and metabolic mechanisms of insulin resistance and beta-cell failure in type 2 diabetes. Nat Rev Mol Cell Biol 9:193205

13. Kelley DE, Goodpaster BH, Storlien L (2002) Muscle triglyceride and insulin resistance. Annu Rev Nutr 22:325-346

14. Ravussin E, Smith SR (2002) Increased fat intake, impaired fat oxidation, and failure of fat cell proliferation result in ectopic fat storage, insulin resistance, and type 2 diabetes mellitus. Ann N Y Acad Sci 967:363-378

15. Lowell BB, Shulman GI (2005) Mitochondrial dysfunction and type 2 diabetes. Science 307:384-387

16. Patti ME, Butte AJ, Crunkhorn S et al (2003) Coordinated reduction of genes of oxidative metabolism in humans with insulin resistance and diabetes: potential role of PGC1 and NRF1. Proc Natl Acad Sci USA 100:8466-8471

17. Morino K, Petersen KF, Dufour S et al (2005) Reduced mitochondrial density and increased IRS-1 serine phosphorylation in muscle of insulin-resistant offspring of type 2 diabetic parents. J Clin Invest 115:3587-3593

18. Kelley DE, He J, Menshikova EV, Ritov VB (2002) Dysfunction of mitochondria in human skeletal muscle in type 2 diabetes. Diabetes 51:2944-2950

19. Toledo FG, Menshikova EV, Ritov VB et al (2007) Effects of physical activity and weight loss on skeletal muscle mitochondria and relationship with glucose control in type 2 diabetes. Diabetes $56: 2142-2147$

20. Menshikova EV, Ritov VB, Toledo FG, Ferrell RE, Goodpaster BH, Kelley DE (2005) Effects of weight loss and physical activity on skeletal muscle mitochondrial function in obesity. Am J Physiol Endocrinol Metab 288:E818-825

21. Eriksson JW (2007) Metabolic stress in insulin's target cells leads to ROS accumulation - a hypothetical common pathway causing insulin resistance. FEBS Lett 581:3734-3742

22. Pieczenik SR, Neustadt J (2007) Mitochondrial dysfunction and molecular pathways of disease. Exp Mol Pathol 83:84-92 
23. Civitarese AE, Ravussin E (2008) Mitochondrial energetics and insulin resistance. Endocrinology 149:950-954

24. Evans JL, Goldfine ID, Maddux BA, Grodsky GM (2002) Oxidative stress and stress-activated signaling pathways: a unifying hypothesis of type 2 diabetes. Endocr Rev 23:599-622

25. Evans JL, Maddux BA, Goldfine ID (2005) The molecular basis for oxidative stress-induced insulin resistance. Antioxid Redox Signal 7:1040-1052

26. Houstis N, Rosen ED, Lander ES (2006) Reactive oxygen species have a causal role in multiple forms of insulin resistance. Nature 440:944-948

27. Evans JL, Goldfine ID, Maddux BA, Grodsky GM (2003) Are oxidative stress-activated signaling pathways mediators of insulin resistance and beta-cell dysfunction? Diabetes 52:1-8

28. Anderson EJ, Lustig ME, Boyle KE et al (2009) Mitochondrial $\mathrm{H}_{2} \mathrm{O}_{2}$ emission and cellular redox state link excess fat intake to insulin resistance in both rodents and humans. J Clin Invest 119:573-581

29. Chung SS, Kim M, Youn BS et al (2009) Glutathione peroxidase 3 mediates the antioxidant effect of peroxisome proliferatoractivated receptor gamma in human skeletal muscle cells. Mol Cell Biol 29:20-30

30. Wilson PW, Paffenbarger RS Jr, Morris JN, Havlik RJ (1986) Assessment methods for physical activity and physical fitness in population studies: report of a NHLBI workshop. Am Heart J 111:1177-1192

31. Dent RM, Penwarden RM, Harris N, Hotz SB (2002) Development and evaluation of patient-centered software for a weightmanagement clinic. Obes Res 10:651-656

32. Ahituv N, Kavaslar N, Schackwitz W et al (2007) Medical sequencing at the extremes of human body mass. Am J Hum Genet 80:779-791

33. DeFronzo RA, Tobin JD, Andres R (1979) Glucose clamp technique: a method for quantifying insulin secretion and resistance. Am J Physiol 237:E214-E223

34. Costford SR, Kavaslar N, Ahituv N et al (2007) Gain-of-function R225W mutation in human AMPKgamma3 causing increased glycogen and decreased triglyceride in skeletal muscle. PLoS ONE. doi:10.1371/journal.pone.0000903
35. Scaduto RC Jr, Grotyohann LW (1999) Measurement of mitochondrial membrane potential using fluorescent rhodamine derivatives. Biophys J 76:469-477

36. Association AD (2008) Standards of medical care in diabetes2008. Diabetes Care 31(Suppl 1):S12-S54

37. Echtay KS, Roussel D, St-Pierre J et al (2002) Superoxide activates mitochondrial uncoupling proteins. Nature 415:9699

38. Echtay KS, Murphy MP, Smith RA, Talbot DA, Brand MD (2002) Superoxide activates mitochondrial uncoupling protein 2 from the matrix side. Studies using targeted antioxidants. J Biol Chem 277:47129-47135

39. Echtay KS, Esteves TC, Pakay JL et al (2003) A signalling role for 4-hydroxy-2-nonenal in regulation of mitochondrial uncoupling. EMBO J 22:4103-4110

40. Kelley DE, Wing R, Buonocore C, Sturis J, Polonsky K, Fitzsimmons M (1993) Relative effects of calorie restriction and weight loss in noninsulin-dependent diabetes mellitus. J Clin Endocrinol Metab 77:1287-1293

41. Goodpaster BH, Kelley DE, Wing RR, Meier A, Thaete FL (1999) Effects of weight loss on regional fat distribution and insulin sensitivity in obesity. Diabetes 48:839-847

42. He J, Kelley DE (2004) Muscle glycogen content in type 2 diabetes mellitus. Am J Physiol Endocrinol Metab 287:E10021007

43. Eriksson J, Franssila-Kallunki A, Ekstrand A et al (1989) Early metabolic defects in persons at increased risk for non-insulindependent diabetes mellitus. N Engl J Med 321:337-343

44. Petersen KF, Befroy D, Dufour S et al (2003) Mitochondrial dysfunction in the elderly: possible role in insulin resistance. Science 300:1140-1142

45. Unoki H, Yamagishi S (2008) Advanced glycation end products and insulin resistance. Curr Pharm Des 14:987-989

46. Schrauwen P, Hesselink MK, Blaak EE et al (2001) Uncoupling protein 3 content is decreased in skeletal muscle of patients with type 2 diabetes. Diabetes 50:2870-2873

47. Hulver MW, Berggren JR, Cortright RN et al (2003) Skeletal muscle lipid metabolism with obesity. Am J Physiol Endocrinol Metab 284:E741-E747 